\title{
Poincaré duality and periodicity
}

\author{
JOHN R KLEIN \\ WILLIAM RICHTER
}

\begin{abstract}
We construct periodic families of Poincaré complexes, partially solving a question of Hodgson, and infinite families of Poincaré complexes whose top cell falls off after one suspension but which fail to embed in a sphere of codimension one. We give a homotopy theoretic description of the four-fold periodicity in knot cobordism.
\end{abstract}

57P10, 57Q45; 55Q25, 55P91

\section{Introduction}

Let $X^{n}$ be a finite oriented Poincaré complex of dimension $\geq 3$. We may suppose (see Wall [23, Theorem 2.4]) that $X=K \cup_{\alpha} D^{n}$ where $K$ is a CW complex of dimension $\leq n-1$, and $\alpha: S^{n-1} \rightarrow K$ is the attaching map for the top cell of $X$. Since $K$ is unique up to homotopy, we call $K$ the spine of $X$. Hodgson [11] posed the question:

Question 1 (Hodgson) Given an $n$-dimensional Poincaré complex $X^{n}$ with spine $K$, is there an $(n+2)$-dimensional Poincaré complex $Y^{n+2}$ with spine $\Sigma K$ ?

By Poincaré duality, the obvious dimension of $Y$ is $n+2$. The answer is often no, for example, the cofibers of the Hopf invariant one maps $\mathbb{C} P^{2}=S^{2} \cup_{\eta} D^{4}$, $\mathbb{H} P^{2}=S^{4} \cup_{v} D^{8}$ and $S^{8} \cup_{\sigma} D^{16}$, whose spines are $S^{2}, S^{4}$ and $S^{8}$ respectively. These examples are generalized by the class pointed out to us by Jim Davis:

Example 1.1 Let $X$ be a connected $4 k$-dimensional Poincaré complex with odd Euler characteristic. Let $K$ be the spine of $X$. Then there is no Poincaré complex $Y$ of dimension $4 k+2$ having spine $\Sigma K$, because the Euler characteristic of $Y$ would have to be odd (since $\chi(X) \equiv \chi(Y) \bmod 2)$. But the Euler characteristic of $Y$ must be even, since its intersection form is skew symmetric. Thus $Y$ can't exist.

The Poincaré complexes $\mathbb{C} P^{2 k}$ and $\mathbb{H} P^{2 k}$ are in this class. The class is closed with respect to taking products. Furthermore, if $X^{4 k}$ is in the above class and $Y^{4 k}$ has even Euler characteristic, then the connected sum $X \# Y$ is in the class. 
Question 1 sometimes has a positive answer: for example, the torus $S^{p} \times S^{q}$ has spine $S^{p} \vee S^{q}$. We formulate a slightly weaker version of Hodgson's question.

Question 2 Given a Poincaré complex $X$ with spine $K$, does there exist an integer $j>0$ and a Poincaré complex $Y$ whose spine is $\Sigma^{j} K$ ?

Adams's Hopf invariant one theorem [1] and $X=S^{8} \cup_{\sigma} D^{16}$ shows that Question 2 can have a negative answer. Question 2 has an affirmative answer for those $X$ whose top cell splits off after a single suspension, and for $j$ even:

Theorem A Let $X^{n}$ be a Poincaré complex with spine $K$ such that the top cell of $X$ splits off after one suspension. Then there exists a Poincaré complex $Y^{n+4}$ whose spine is $\Sigma^{2} K$ and whose top cell splits off after one suspension.

If a Poincaré complex embeds in codimension one, then its top cell splits off after one suspension: if $X^{n} \subset S^{n+1}$ is a codimension one Poincare embedding, then the Pontryagin-Thom construction gives a degree one map $S^{n+1} \rightarrow \Sigma X$ which splits off the top cell of $X$. We answer Question 1 for this class of Poincaré complexes:

Theorem B Suppose $X$ as above has spine $K$. Then $\Sigma K$ is the spine of a Poincaré complex $Y$, and $Y$ has a codimension one Poincaré embedding in $S^{n+3}$.

The hypothesis of Theorem B implies that of Theorem A. We show that the converse need not hold: we will construct infinite families of Poincaré complexes whose top cell falls off after one suspension, but which fail to Poincaré embed in a sphere in codimension one. Note however by a result of Browder [6] that any such example must necessarily embed in codimension two.

See Section 2 for the definition of Whitehead products, and let $x, y: S^{n} \rightarrow S^{n} \vee S^{n}$ be the inclusions into each summand. Our first infinite family is given by the "Kervaire" PL manifolds (see Kosinski [15, page 120, Corollary 4.7]).

Proposition $\mathbf{C}$ For any odd whole number $n \neq 1,3,7$, the mapping cone of the map

$$
[x, x]+[y, x]+[y, y]: S^{2 n-1} \rightarrow S^{n} \vee S^{n}
$$

is a $2 n$-dimensional Poincaré complex which does not embed in codimension one but whose top cell falls off after one suspension. 
We assume the reader is familiar with Toda's book [22]. Recall that at the prime 2 one has an EHP-sequence with connecting map $P: \pi_{*+2}\left(S^{2 n+1}\right) \rightarrow \pi_{*}\left(S^{n}\right)$. Then $P$ is represented by a map $\Omega^{2} S^{2 n+1} \rightarrow S^{n}$ after localizing at 2 , so given a map $f: S^{j+2} \rightarrow$ $S^{2 n+1}$, one may consider the 2-local map $P(f): S^{j} \rightarrow S^{n}$. The following result provides criteria for constructing infinite families of examples.

Proposition D Given a map $\alpha: S^{p+q+1} \rightarrow S^{2 q+1}$ of order $2^{r+1}$, with $q$ even, $p \geq 2 q$ and $r>1$, let $A$ be the cofiber of the map

$$
[y, x]+y P(\alpha): S^{p+q-1} \rightarrow S^{p} \vee S^{q} .
$$

If $2^{r}$ kills the image of $E: \pi_{p}\left(S^{q}\right) \rightarrow \pi_{p+1}\left(S^{q+1}\right)$, then $A$ is a $(p+q)$-dimensional Poincaré complex whose top cell falls off after one suspension. However, $A$ does not embed in codimension one.

The Poincaré complex $A^{19}$ with attaching map $[y, x]+y P\left(E^{5} \sigma\right): S^{18} \rightarrow S^{13} \vee S^{6}$ is an example of Proposition D, as Toda's first table [22, page 186] shows the image of $E: \pi_{13}\left(S^{6}\right) \rightarrow \pi_{14}\left(S^{7}\right)$ has order at most 4 , whereas $E^{5} \sigma \in \pi_{20}\left(S^{13}\right)$ has order 16. Recall the Adams self-map $W: M_{16 \iota}^{n+8} \rightarrow M_{16 \iota}^{n}$ of the Moore space which exists for $n>10$ for stability reasons [2, Lemma 12.5]. We also need an unstable Adams self-map:

Lemma 1.2 For $n \geq 9$, there exists a map $V: M_{8 \iota}^{n+8} \rightarrow M_{8 \iota}^{n}$ so that the composite

$$
S^{n+7} \stackrel{i}{\rightarrow} M_{8 \iota}^{n+8} \stackrel{V}{\longrightarrow} M_{8 \iota}^{n} \stackrel{j}{\longrightarrow} S^{n}
$$

is homotopic to $2 \sigma$.

We will use complex $K$-theory to give a simple proof of the following result, known to Mahowald [18, Theorem 1.5], and possibly also known to Barratt and Toda.

Theorem $\mathbf{E}$ (1) There exist homotopy classes $N_{k} \in \pi_{8 k}\left(S^{5}\right)$ of order 8 , where $N_{1}=v$, and for $k>1, N_{k}$ is the composite

$$
S^{8 k} \stackrel{i}{\longrightarrow} M_{8 \iota}^{8 k+1} \stackrel{V^{\circ(k-1)}}{\longrightarrow} M_{8 \iota}^{9} \stackrel{\nu^{\sharp}}{\longrightarrow} S^{5},
$$

where $v^{\sharp}$ is given by a nullhomotopy of $8 v: S^{8} \rightarrow S^{5}$.

(2) There exist homotopy classes $S_{k} \in \pi_{8 k}\left(S^{9}\right)$ of order 16, where $S_{2}=\sigma$, and for $k>2, S_{k}$ is the composite

$$
S^{8 k \stackrel{i}{\rightarrow}} M_{16 \iota}^{8 k+1} \stackrel{W^{\circ(k-2)}}{\longrightarrow} M_{16 \iota}^{17} \stackrel{\sigma^{\sharp}}{\longrightarrow} S^{9},
$$

where $\sigma^{\sharp}$ is given by a nullhomotopy of $16 \sigma$. 
Note that Mahowald has a powerful framework that explains and extends these elements [18, Theorem 1.5]. Using these two families and Proposition D, we obtain

Corollary $\mathbf{F}$ The cofibers of the maps

$$
\begin{aligned}
& {[y, x]+y P\left(S_{k}\right): S^{8 k-2} \rightarrow S^{8 k-5} \vee S^{4}} \\
& {[y, x]+y P\left(N_{k}\right): S^{8 k-2} \rightarrow S^{8 k-3} \vee S^{2}}
\end{aligned}
$$

are $(8 k-1)$-dimensional Poincaré complexes whose top cells fall off after one suspension, but do not embed in codimension one.

(Here, $x: S^{p} \rightarrow S^{p} \vee S^{q}$ and $y: S^{q} \rightarrow S^{p} \vee S^{q}$ denote the evident inclusions.)

The surgery exact sequence shows the above examples have the homotopy type of smooth manifolds. Other Poincaré complexes whose top cell falls off after one suspension are provided by closing up Seifert surfaces of high dimensional knots. However, we lack criteria for deciding when these fail to embed in codimension one.

In Section 2 we review Boardman and Steer's work on Hopf invariants and prove Theorem A. We give a criterion for Poincaré duality when the top cell splits off after one suspension. In Section 3 we prove Theorem B, and in Section 4, Propositions D and C. In Section 5 we discuss Toda brackets and prove Theorem E and Corollary F. We explain in Section 6 how a variant of Theorem A gives rise to a periodicity operator for knot theory, inducing the four-fold periodicity of the knot cobordism groups. In Section 7, we discuss our linear notion of periodicity, and explain some exponential periodicity of Mahowald.

Acknowledgements The authors are grateful to Mark Mahowald for his insight and guidance. We are also indebted Brayton Gray and Bob Bruner for helping us understand how Adams's work [2] proves Theorem E. The first author wishes to thank Andrew Ranicki for help with the surgery theory literature, Diarmuid Crowley for discussions in connection with Proposition D, and Matthias Kreck and Peter Teichner for pointing out that $\Sigma \mathbb{R} P^{2}$ is the spine of $\mathrm{SU}(3) / \mathrm{SO}(3)$.

The first author was partially supported by the National Science Foundation.

\section{Poincaré duality and Hopf invariants}

The spaces in this paper are assumed to have the homotopy type of CW complexes. Basepoints are always assumed to be non-degenerate. If $X$ is a based space then $\Sigma X$ denotes its reduced suspension, and $\Omega X$ denotes its based loop space. The smash 
product of based spaces $A$ and $B$ is denoted $A \wedge B$. Let $[A, B]$ denote the (based) homotopy classes of maps from $A$ to $B$, and let $\{A, B\}$ be the abelian group of stable homotopy classes of maps from $A$ to $B$.

See Wall [23] for the definition of a Poincaré complex. We consider only finite oriented Poincaré complexes. If $X$ is an $n$-dimensional Poincaré complex, there is a fundamental class $[X] \in H_{n}(X)$ giving a cap product isomorphism

$$
\cap[X]: H^{k}(X) \stackrel{\cong}{\rightarrow} H_{n-k}(X) \quad \text { for all integers } k \text {. }
$$

If $X$ is a 1-connected finite complex with a class $[X] \in H_{n}(X)$ satisfying (1), then $X$ is a Poincaré complex. Similar remarks hold for Poincaré pairs.

If $A$ and $B$ have the homotopy type of finite complexes, then a (stable) map $d: S^{n} \rightarrow$ $A \wedge B$ is an $S$-duality map if and only if the slant product homomorphism

$$
/ d_{*}\left[S^{n}\right]: \widetilde{H}^{*}(A) \rightarrow \widetilde{H}_{n-*}(B)
$$

is an isomorphism in all degrees. Here $\tilde{H}_{*}$ means reduced singular homology, and $\left[S^{n}\right] \in \widetilde{H}_{n}\left(S^{n}\right)$ denotes the generator.

We rely on Boardman and Steer's treatment of Hopf invariants in [4], much as we did earlier [20]. Let $B$ be a based space. The suspension map $E: B \rightarrow \Omega \Sigma B$ is adjoint to the identity. The James Hopf invariant $H: \Omega \Sigma B \rightarrow \Omega \Sigma(B \wedge B)$ is a natural map [4, 3.10] with $H \circ E$ canonically null homotopic. (We will not need this, but

$$
B \stackrel{E}{\rightarrow} \Omega \Sigma B \stackrel{H}{\rightarrow} \Omega \Sigma(B \wedge B)
$$

is a metastable homotopy fiber sequence.) $H$ gives a natural map $H:[\Sigma A, \Sigma B] \rightarrow$ $[\Sigma A, \Sigma B \wedge B]$. The Hopf invariant $\lambda:[\Sigma A, \Sigma B] \rightarrow\left[\Sigma^{2} A, \Sigma B \wedge \Sigma B\right]$ is the natural map $[4,3.15]$ given by suspending $H$. Boardman and Steer stress the Cartan formula [4, Theorem 3.15, Definition 2.1]:

(2) $\lambda(f+g)=\lambda(f)+f \cdot g+\lambda(g) \in\left[\Sigma^{2} A, \Sigma B \wedge \Sigma B\right], \quad$ for $f, g: \Sigma A \rightarrow \Sigma B$,

where the cup product term $f \cdot g$ means the composite

$$
\Sigma^{2} A \stackrel{\Sigma^{2} \Delta_{A}}{\longrightarrow} \Sigma^{2}(A \wedge A) \stackrel{\text { shuffle }}{\longrightarrow} \Sigma A \wedge \Sigma A \stackrel{f \wedge g}{\longrightarrow} \Sigma B \wedge \Sigma B .
$$

Following [4], we use right suspensions, so $\Sigma A:=A \wedge S^{1}$, and suppress shuffle maps. Note that by shuffling the suspension coordinates around we can show that

$$
f \cdot g=0 \in\left[\Sigma^{2} A, \Sigma B \wedge \Sigma B\right] \quad \text { if } \Sigma f=0 \in\left[\Sigma^{2} A, \Sigma^{2} B\right]
$$


Let $\tau_{K}: K \wedge K \rightarrow K \wedge K$ be the twist map (which switches factors). The proof of [4, Theorem 3.17] (which assumed $B$ is a suspension) generalizes to prove

$$
(f \wedge f) \circ \Sigma^{2} \Delta_{A}=\left(1-\tau_{\Sigma B}\right) \circ \lambda(f)+\Sigma^{2} \Delta_{B} \circ \Sigma f \in\left[\Sigma^{2} A, \Sigma B \wedge \Sigma B\right] .
$$

As Boardman and Steer stress, the following diagrams commute up to homotopy, for maps $f, g: \Sigma A \rightarrow \Sigma B$, because the twist $\tau_{S^{1}}$ on $S^{1} \wedge S^{1}$ has degree -1 .
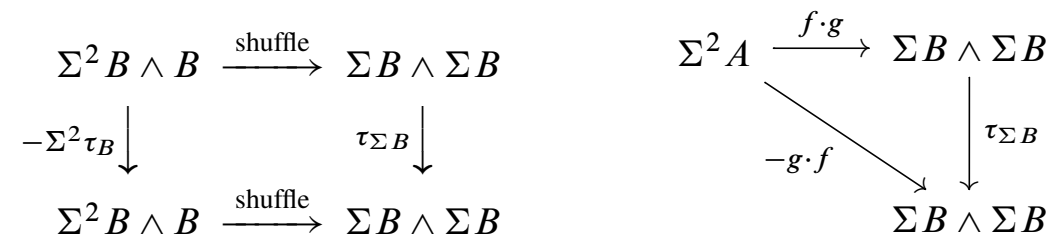

For maps $f: \Sigma P \rightarrow X$ and $g: \Sigma Q \rightarrow X$, the Whitehead product [4, 4.2] [f,g]: $\Sigma P \wedge$ $Q \rightarrow X$ is defined as the unique homotopy class so that the composite

$$
\Sigma(P \times Q) \stackrel{\Sigma \pi_{12}}{\longrightarrow} \Sigma P \wedge Q \stackrel{[f, g]}{\longrightarrow} X
$$

is the commutator $\left(f \circ \Sigma \pi_{1}, g \circ \Sigma \pi_{2}\right)$. We extend [4, Theorem 4.6] to the case when $P$ and $Q$ are not required to be suspensions:

Lemma 2.1 Given maps $f: \Sigma P \rightarrow \Sigma B$ and $g: \Sigma Q \rightarrow \Sigma B$, the Whitehead product $[f, g]: \Sigma P \wedge Q \rightarrow \Sigma X$ has Hopf invariant

$$
\lambda([f, g])=\left(1+\tau_{\Sigma X}\right) \circ(f \wedge g): \Sigma^{2} P \wedge Q \rightarrow \Sigma X \wedge \Sigma X .
$$

In particular, for the Whitehead product $[\iota, \iota]: \Sigma X \wedge X \rightarrow \Sigma X$, we have

$$
\lambda([\iota, \iota])=1+\tau_{\Sigma X}: \Sigma^{2} X \wedge X \rightarrow \Sigma X \wedge \Sigma X .
$$

Proof The map $\Sigma \pi_{12}: \Sigma(P \times Q) \rightarrow \Sigma(P \wedge Q)$ is a stably split surjection, so it suffices to prove (6) pulled back to $\Sigma(P \times Q)$. Write $f_{1}=f \circ \Sigma \pi_{1}$ and $g=g \circ$ $\Sigma \pi_{2}: \Sigma P \times Q \rightarrow \Sigma X$. By definition, $[f, g] \circ \Sigma \pi_{12}=\left(f_{1}, g_{2}\right) \in[\Sigma(P \times Q), \Sigma X]$. Write $\left(f_{1}, g_{2}\right)=F-G$, where $F=f_{1}+g_{2}$ and $G=g_{2}+f_{1}$. Then $F=\left(f_{1}, g_{2}\right)+G$. By (3), the cup product $\left(f_{1}, g_{2}\right) \cdot G$ is nullhomotopic, because $\Sigma\left(f_{1}, g_{2}\right)$ is nullhomotopic. Thus

$$
\lambda\left(f_{1}\right)+f_{1} \cdot g_{2}+\lambda\left(g_{2}\right)=\lambda\left(\left(f_{1}, g_{2}\right)\right)+\lambda\left(g_{2}\right)+g_{2} \cdot f_{1}+\lambda\left(f_{1}\right)
$$

by the Cartan formula (2). By (5) and $f_{1} \cdot g_{2}$ being a suspension, we have

$$
\lambda\left(\left(f_{1}, g_{2}\right)\right)=f_{1} \cdot g_{2}-g_{2} \cdot f_{1}=\left(1+\tau_{\Sigma X}\right) \circ f_{1} \cdot g_{2}=\left(1+\tau_{\Sigma X}\right) \circ(f \wedge g) \circ \Sigma^{2} \pi_{12} .
$$


Given a map $f: \Sigma A \rightarrow \Sigma B$ with cofiber $X$, the diagram

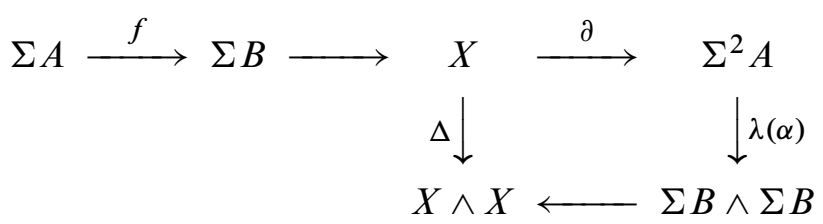

is homotopy commutative [4, Theorem 5.14]. This immediately implies the following.

Proposition 2.2 Let $L$ be a connected finite complex of dimension $\leq n-3$, and $\alpha: S^{n-1} \rightarrow \Sigma L$ a based map with cofiber $X$. Then $X$ is a Poincaré complex if and only if $\lambda(\alpha): S^{n} \rightarrow \Sigma L \wedge \Sigma L$ is an $S$-duality map.

Suppose the cofiber of the based map $\alpha: S^{n-1} \rightarrow K$ is a Poincaré complex $X$ whose top cell splits off after one suspension, so we have a degree one map $\rho: S^{n+1} \rightarrow \Sigma X$. Then $\Sigma i \vee \rho: \Sigma K \vee S^{n+1} \rightarrow \Sigma X$ is a homotopy equivalence and defines a map $f: \Sigma X \rightarrow \Sigma K$ so that the composite $f \circ \Sigma i: \Sigma K \rightarrow \Sigma K$ is homotopic to the identity, and the composite $f \circ \rho: S^{n+1} \rightarrow \Sigma K$ is nullhomotopic.

Proposition 2.3 If $X$ is a Poincaré complex, the composite is an $S$-duality map:

$$
S^{n+2} \stackrel{\Sigma \rho}{\longrightarrow} \Sigma^{2} X \stackrel{\lambda(f)}{\longrightarrow} \Sigma K \wedge \Sigma K \stackrel{1-\tau_{\Sigma K}}{\longrightarrow} \Sigma K \wedge \Sigma K .
$$

Proof Applying the symmetrization formula (4) to $f: \Sigma X \rightarrow \Sigma K$ gives

$$
(f \wedge f) \circ \Sigma^{2} \Delta_{X}=\left(1-\tau_{\Sigma K}\right) \circ \lambda(f)+\Sigma^{2} \Delta_{K} \circ \Sigma f \in\left[\Sigma^{2} X, \Sigma K \wedge \Sigma K\right] .
$$

Since $f \circ \rho$ is nullhomotopic, right composition with $\Sigma \rho$ gives

$$
(f \wedge f) \circ \Sigma^{2} \Delta_{X} \circ \Sigma \rho=\left(1-\tau_{\Sigma K}\right) \circ \lambda(f) \circ \Sigma \rho \in\left[S^{n+2}, \Sigma K \wedge \Sigma K\right] .
$$

Relative Poincaré duality is given by a map $\widetilde{\Delta}: X \rightarrow K \wedge K$, so the composite $\Sigma \tilde{\Delta} \circ \rho$ is an $S$-duality map. But $\Delta_{X}: X \rightarrow X \wedge X$ is homotopic to the composite

$$
\Delta_{X}: X \stackrel{\tilde{\Delta}}{\longrightarrow} K \wedge K \stackrel{i \wedge i}{\longrightarrow} X \wedge X .
$$

Thus $(f \wedge f) \circ \Sigma^{2} \Delta_{X}$ is homotopic to $\Sigma^{2} \tilde{\Delta}$. Hence $(f \wedge f) \circ \Sigma^{2} \Delta_{X} \circ \Sigma \rho$ is homotopic to $\Sigma^{2} \tilde{\Delta} \circ \Sigma \rho$, which is an $S$-duality map.

Proof of Theorem A As above, let $X^{n}=K \cup_{\alpha} D^{n}$ be a Poincaré complex whose top cell splits off after one suspension by a degree one map $\rho: S^{n+1} \rightarrow \Sigma X$. Suspend twice the $S$-duality map of Proposition 2.3. By using (5), we see the composition

$$
S^{n+4} \stackrel{\Sigma^{3} \rho}{\longrightarrow} \Sigma^{4} X \stackrel{\Sigma^{2} \lambda(f)}{\longrightarrow} \Sigma^{2} K \wedge \Sigma^{2} K \stackrel{1+\tau_{\Sigma^{2} K}}{\longrightarrow} \Sigma^{2} K \wedge \Sigma^{2} K
$$


is an $S$-duality map. Define $\beta: S^{n+3} \rightarrow \Sigma^{2} K$ as the composition

$$
\beta: S^{n+3} \stackrel{\Sigma^{2} \rho}{\longrightarrow} \Sigma^{3} X \stackrel{\Sigma \lambda(f)}{\longrightarrow} \Sigma(\Sigma K \wedge \Sigma K) \stackrel{[\iota, l]}{\longrightarrow} \Sigma^{2} K .
$$

Let $Y$ be the cofiber of $\beta$. Lemma 2.1 and naturality shows $\lambda(\beta)$ is composition (8). Therefore $\lambda(\beta)$ an $S$-duality map. By Proposition 2.2, $Y$ is an $(n+4)$-dimensional Poincaré complex. Clearly the top cell splits off $Y^{n+4}$ after one suspension, because the suspension of a Whitehead product is nullhomotopic.

Note that in above proof we could have tried unsuccessfully to construct an $(n+2)-$ dimensional Poincaré complex as the cofiber $Z$ of the composition

$$
\gamma: S^{n+1} \stackrel{\rho}{\rightarrow} \Sigma X \stackrel{H(f)}{\longrightarrow} \Sigma(K \wedge K) \stackrel{[\iota, l]}{\longrightarrow} \Sigma K .
$$

The composition formula [4, Theorem 3.16] calculates $\lambda(\gamma)$ to be the composition

$$
S^{n+2} \stackrel{\Sigma \rho}{\longrightarrow} \Sigma^{2} X \stackrel{\lambda(f)}{\longrightarrow} \Sigma K \wedge \Sigma K \stackrel{1+\tau_{\Sigma K}}{\longrightarrow} \Sigma K \wedge \Sigma K .
$$

Since we have $1+\tau_{K}$ instead of $1-\tau_{K}$, we don't know that $\lambda(\beta)$ is an $S$-duality map, so we can't conclude that $Z$ is an $(n+2)$-dimensional Poincaré complex.

\section{Periodicity in the codimension one case}

In this section we will prove Theorem B, solving Question 1 for the class of Poincaré complexes having codimension one embeddings in the sphere. For the definition of Poincaré embedding see, for example, Klein [14]. Let $X^{n}$ be a connected oriented $n$-dimensional Poincaré complex which is Poincaré embedded in $S^{n+1}$. Theorem B is a direct consequence of the following.

Proposition 3.1 If $L$ denotes the spine of $X$, then $\Sigma L$ is the spine of a Poincare complex $Y$, and $Y$ has a codimension one Poincaré embedding in $S^{n+3}$.

Proof The proof will rely on the decompression construction of [14, Section 2.3]. By Spanier-Whitehead duality, the complement of $X \subset S^{n+1}$ has two components, call them $M$ and $W$. The normal data define inclusions $X \rightarrow M$ and $X \rightarrow W$ which form Poincaré pairs of dimension $n+1$. Then we have a homotopy pushout diagram

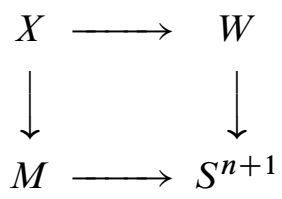


which gives a Poincaré embedding of $M$ with complement $W$.

The fiberwise suspension $S_{M} X$ of $X$ over $M$ is the double mapping cylinder $S_{M} X=$ $M \times 0 \cup X \times[0,1] \cup M \times 1$, together with the evident map $S_{M} X \rightarrow M$ [14, page 609]. Note that $M \times 0$ provides a section $M \rightarrow S_{M} X$, and the map $X \rightarrow W$ induces a map $S_{M} X \rightarrow \Sigma W$ given by collapsing each copy of $M$ to a point. Then the homotopy pushout diagram

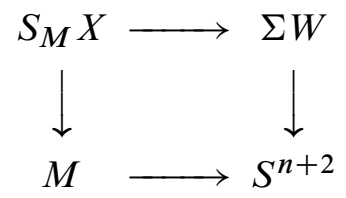

is a Poincaré embedding of $M$ in $S^{n+2}$ with complement $\Sigma W$. This is the decompression of $M$ in $S^{n+2}$, which is well understood if $M$ is a closed submanifold of $S^{n+1}$, and $X$ is the sphere bundle of the normal bundle. Reversing the roles of $M$ and $\Sigma W$, we decompress once more to get a Poincaré embedding

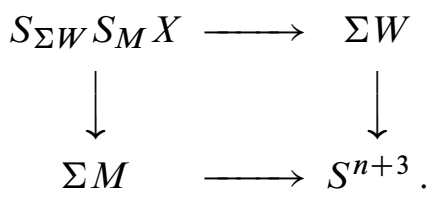

Set $Y=S_{\Sigma W} S_{M} X$, and note that the maps $Y \rightarrow \Sigma M$ and $Y \rightarrow \Sigma W$ have sections. The sum of these gives a map $\Sigma M \vee \Sigma W \rightarrow Y$ which is seen to be $(n+1)$-connected by application of the Mayer-Vietoris sequence to the diagram. The relative Hurewicz theorem shows that $Y$ is obtained from $\Sigma M \vee \Sigma W$ by attaching an $(n+2)-$ cell. So $\Sigma M \vee \Sigma W=\Sigma(M \vee W)$ is the spine of $Y$.

For a harder proof similar to the proof of Theorem A, define $Y$ as the cofiber of the composite $S^{n+1} \stackrel{\mathcal{D}}{\rightarrow} \Sigma M \wedge W \stackrel{[y, x]}{\longrightarrow} \Sigma M \vee \Sigma W$ (using the $S$-duality map $\mathcal{D}$ of Lemma 4.1 below), whose Hopf invariant is an $S$-duality map by Lemma 2.1. $Y$ is a Poincaré complex by Proposition 2.2. There are obvious maps $Y \rightarrow \Sigma M$ and $Y \rightarrow \Sigma W$, which one can show determines a Poincaré embedding in $S^{n+2}$.

\section{Proof of Propositions D and C}

Lemma 4.1 Let $A=\left(S^{p} \vee S^{q}\right) \cup_{\alpha} D^{p+q}$ be a three cell complex satisfying Poincaré duality, where $p, q>1$. If $A$ has a Poincare embedding in $S^{p+q+1}$, then there is a homotopy equivalence $A \simeq S^{p} \times S^{q}$. 
Proof Given a codimension one Poincaré embedding of $A$, its complement has two components $M$ and $W$. We have a homotopy pushout and a splitting after one suspension [20]

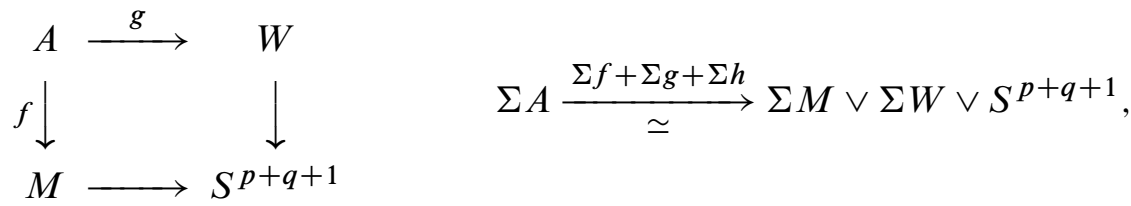

where $h: A \rightarrow S^{p+q}$ is the pinch onto the top cell. By the van Kampen theorem, $M$ and $W$ are 1-connected. We will show that $M$ and $W$ are homotopy equivalent to the spheres $S^{p}$ and $S^{q}$, and that the map $F=f \times g: A \rightarrow M \times W$ is a homotopy equivalence. Let $A_{0}=S^{p} \vee S^{q}$. Since $A_{0}$ is a co-H space, the restriction of $F$ to $A_{0}$ factors up to homotopy through the wedge by a map $F_{0}=x f+y g: A_{0} \rightarrow M \vee W$. By the stable splitting, $F_{0}$ is a homotopy equivalence by the Whitehead theorem.

Assume $p \neq q$. Since

$$
\mathbb{Z} \cong H_{p}\left(A_{0}\right) \underset{\cong}{\stackrel{F_{*}}{\longrightarrow}} H_{p}(M) \oplus H_{p}(W),
$$

one of the summands is 0 . Assume $H_{p}(M) \cong \mathbb{Z}$ and $H_{p}(W)=0$. By Alexander duality, $H_{q}(M)=0$ and $H_{q}(W) \cong \mathbb{Z}$. Since $F_{*}$ is an isomorphism, all other reduced homology groups of $M$ and $W$ vanish. Thus we have homotopy equivalences $M \simeq S^{p}$ and $W \simeq S^{q}$.

Assume $p=q$. Now $\mathbb{Z} \oplus \mathbb{Z} \cong H_{p}\left(A_{0}\right) \cong H_{p}(M) \oplus H_{p}(W)$, and all other reduced homology groups of $M$ and $W$ vanish. By Alexander duality, neither $M$ nor $W$ is contractible, so we have $\mathbb{Z} \cong H_{p}(M) \cong H_{p}(W)$, and again $M \simeq S^{p}$ and $W \simeq S^{q}$.

Thus we have shown that $F$ is a homology isomorphism except in degree $p+q$, where $F$ is degree one because (see Klein [13, Proposition 2.3] and [20, Section 2]) Alexander duality is induced by the $S$-duality map

$$
\mathcal{D}: S^{p+q+1} \stackrel{\text { collapse }}{\longrightarrow} \Sigma A \stackrel{\Sigma \Delta}{\longrightarrow} \Sigma A \wedge A \rightarrow \Sigma M \wedge W \simeq S^{p+q+1} .
$$

Therefore, composing $F$ with the homotopy equivalences $M \simeq S^{p}$ and $W \simeq S^{q}$ gives a homotopy equivalence $A \rightarrow S^{q} \times S^{p}$.

Proof of Proposition D The space $A$ is a Poincaré complex of dimension $p+q$ since the cup product structure on $A$ is determined by the term $[y, x]$ appearing in the attaching map, that is, the cohomology ring of $A$ is just the cohomology ring of $S^{p} \times S^{q}$. 
In order to prove that there does not exist a codimension one Poincaré embedding, using Lemma 4.1 it will suffice to show that there is no map $A \rightarrow S^{q}$ which has degree one on the $q$-cell. Assume that such a map exists. By the cofibration sequence

$$
S^{p+q-1} \stackrel{[y, x]+y P(\alpha)}{\longrightarrow} S^{p} \vee S^{q} \rightarrow A,
$$

there must be a map $f: S^{p} \rightarrow S^{q}$ so the following composite is nullhomotopic:

$$
S^{p+q-1} \stackrel{[y, x]+y P(\alpha)}{\longrightarrow} S^{p} \vee S^{q} \stackrel{f \vee 1}{\longrightarrow} S^{q} .
$$

This composite is $[\iota, f]+P(\alpha) \in \pi_{p+q-1}\left(S^{q}\right)$, by naturality of Whitehead products. But $[\iota, f]$ is homotopic to the composite

$$
S^{p+q-1} \stackrel{\Sigma^{q-1} f}{\longrightarrow} S^{2 q-1} \stackrel{[\iota, l]}{\longrightarrow} S^{q},
$$

by the Barcus-Barratt theorem [3] and the fact (see Cohen [9]) that at the prime 2, all higher Whitehead products vanish. Now $[\iota, \iota]: S^{2 q-1} \rightarrow S^{q}$ is homotopic (see Whitehead [25]) to the composite $P \circ E^{2}: S^{2 q-1} \rightarrow S^{q}$. Thus $[\iota, f]=P\left(\Sigma^{q+1} f\right) \in$ $\pi_{p+q-1}\left(S^{q}\right)$. Since our composite (10) is nullhomotopic, we have $P\left(\Sigma^{q+1} f+\alpha\right)=$ $0 \in \pi_{p+q-1}\left(S^{q}\right)$. By the exactness of the EHP sequence, $\Sigma^{q+1} f+\alpha=H(\beta) \in$ $\pi_{p+q-1}\left(S^{2 q+1}\right)$ for some $\beta \in \pi_{p+q-1}\left(S^{q+1}\right)$. By James's theorem (see Cohen [9]), $2 H(\beta)=0$, since $q$ is even, so

$$
2 \alpha+2 E^{q+1} f=0 \in \pi_{p+q+1}\left(S^{2 q+1}\right) .
$$

Multiplying this equation above by $2^{r-1}$ gives the contradiction $2^{r} \alpha=0$.

Proof of Proposition C Recall that $n$ is an odd integer $\neq 1,3,7$. Let $A$ be the cofiber of the map appearing in the statement of Proposition C. Then the top cell of $A$ falls off after one suspension since the attaching map is a sum of Whitehead products. Furthermore, $A$ is a $2 n$-dimensional Poincaré complex since the cup product structure on $A$ is determined by the term $[y, x]$ appearing in the attaching map, that is, $H^{*}(A) \cong H^{*}\left(S^{n} \times S^{n}\right)$.

Assume $A$ has a Poincaré embedding in $S^{2 n+1}$. By Lemma 4.1, there is a homotopy equivalence $A \simeq S^{n} \times S^{n}$. The projection of this equivalence onto the first factor is a map $f: A \rightarrow S^{n}$ such that $f^{*}(x)$ extends to a basis of $H^{n}(A)$, where $x \in H^{n}\left(S^{n}\right)$ is a generator. Thus the restriction of $f$ to the $n$-skeleton $A_{0}$ is a map of the form $a \iota \vee b \iota: S^{n} \vee S^{n} \rightarrow S^{n}$, with $a$ and $b$ relatively prime. By the cofibration sequence defining $A$, the composite

$$
S^{2 n-1} \stackrel{[x, x]+[y, x]+[y, y]}{\longrightarrow} S^{n} \vee S^{n} \stackrel{a \iota \vee b \iota}{\longrightarrow} S^{n}
$$


is nullhomotopic. By naturality of Whitehead products, this composite is

$$
[a \iota, a \iota]+[b \iota, a \iota]+[b \iota, b \iota]=[\iota, \iota] a^{2}+[\iota, \iota] a b+[\iota, \iota] b^{2}=[\iota, \iota](a+a b+b),
$$

using the left-distributivity of composition. Since $n$ is odd, $2[\iota, \iota]=0$ (see Cohen [9]), but since $n \neq 1,3,7$, the Hopf invariant one theorem (see Adams [1]) implies that $[\iota, \iota] \neq 0$. Hence $[\iota, \iota]$ has order 2 . Thus $a+a b+b \equiv 0(\bmod 2)$, so $(a+1)(b+1) \equiv$ $1(\bmod 2)$. Thus both $a$ and $b$ are even. But $a$ and $b$ are relatively prime, so we have a contradiction. Hence $A$ does not embed in codimension one.

\section{Proof of Theorem E and Corollary $F$}

The cofiber of a map $\gamma: S^{u-1} \rightarrow S^{v}$ is called the Moore space $M_{\gamma}^{u}$, and the cofiber $Y \cup_{g} C X$ of a map $g: X \rightarrow Y$ will also be written $M_{g}$. The generator of $\pi_{7}^{s} \cong \mathbb{Z} / 16$ is $\sigma \in \pi_{15}\left(S^{8}\right)$, and by Toda [22, Proposition 5.15] $2 \sigma \in \pi_{7}^{s}$ desuspends to the generator $\sigma^{\prime} \in \pi_{14}\left(S^{7}\right) \cong \mathbb{Z} / 8$. Let $\tau: \Sigma W \rightarrow Z$ belong to the Toda bracket $\{h, g, f\}$ of the sequence

$$
W \stackrel{f}{\rightarrow} X \stackrel{g}{\rightarrow} Y \stackrel{h}{\rightarrow} Z .
$$

The map $\tau$ is defined as a composite

$$
S W \stackrel{f_{b}}{\rightarrow} M_{g} \stackrel{h^{\sharp}}{\rightarrow} Z
$$

defined using nullhomotopies of $g f$ and $h g$. The diagram

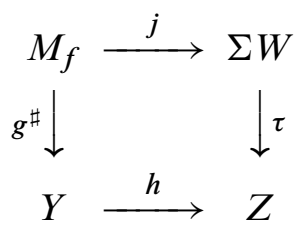

is homotopy commutative (see Adams [2, Diagram (5.1)]), where $g^{\sharp:} M_{f} \rightarrow Y$ is defined using the same nullhomotopy of $g f$. Suppose the Toda bracket $\{h, g, f\}$ contains 0 . Then some $\tau=h^{\sharp} \circ f_{\mathrm{b}}$ is nullhomotopic. By diagram (11), the composite

$$
M_{f} \stackrel{g^{\sharp}}{\rightarrow} Y \stackrel{h}{\rightarrow} Z
$$

is nullhomotopic, and so defines a map $V=\left(g^{\sharp}\right)_{b}: \Sigma M_{f} \rightarrow M_{h}$. Thus the composite

$$
\Sigma X \stackrel{\Sigma i}{\rightarrow} \Sigma M_{f} \stackrel{V}{\rightarrow} M_{h} \stackrel{j}{\rightarrow} \Sigma Y
$$

is homotopic to $\Sigma g$. We now have the following. 
Proof of Lemma 1.2 Toda [22, Cororollary 3.7] shows the Toda bracket $\left\{8 \iota, E \sigma^{\prime}, 8 \iota\right\}$ of the sequence

$$
S^{8} \stackrel{8 \iota}{\longleftarrow} S^{8} \stackrel{E \sigma^{\prime}}{\longleftarrow} S^{15} \stackrel{8 \iota}{\longleftarrow} S^{15}
$$

contains 0 . Thus we have a map

$$
M_{8 \iota}^{17} \stackrel{V}{\rightarrow} M_{8 \iota}^{9}
$$

so that composite $j \circ V \circ i$ is homotopic to $E^{2} \sigma^{\prime}$, which is homotopic to $2 \sigma \in \pi_{16}\left(S^{9}\right)$. The other maps with $n>9$ are obtained by suspending $V$.

Proof of Theorem $\mathbf{E}$ For the $S_{k}$ family, we require only that the Adams self-map is a $K$-theory isomorphism [2].

Recall the Toda bracket $\{h, g, f\}$ of a sequence $W \stackrel{f}{\rightarrow} X \stackrel{g}{\rightarrow} Y \stackrel{h}{\rightarrow} Z$. Any map $\tau: \Sigma W \rightarrow Z$ making diagram (11) homotopy commute must belong to $\{h, g, f\}:$ we know some element of the Toda bracket $\tau_{0} \in\{h, g, f\}$ makes diagram (11) homotopy commute, and by exactness of the cofibration sequence

$$
M_{f} \rightarrow \Sigma W \stackrel{\Sigma f}{\longrightarrow} \Sigma X
$$

we know that $\tau=\tau_{0}+p \circ \Sigma f \in[\Sigma W, Z]$, for some element $p \in[\Sigma X, Z]$. But $p \circ \Sigma f \in[\Sigma W, Z]$ belongs to the indeterminacy of $\{h, g, f\}$. Hence $\tau \in\{h, g, f\}$.

By Bott periodicity, $\pi_{2 n}(B U) \cong \mathbb{Z}$ and $\pi_{2 n-1}(B U)=0$, for $n>0$. The generator $\zeta_{n}: S^{2 n} \rightarrow B U$ is the $n$-fold exterior power of the bottom generator $\zeta_{1} \in B U$, which comes of course from $S^{2}=\mathbb{C} P^{1} \subset \mathbb{C} P^{\infty}=B U(1) \subset B U$.

Given $f \in \pi_{2 m-1} S^{2 k}$ with $q f=0$, consider the Toda bracket $\left\{\zeta_{k}, f, q \iota\right\} \subset \mathbb{Z}$ of the sequence

$$
B U \stackrel{\zeta_{k}}{\leftarrow} S^{2 k} \stackrel{f}{\leftarrow} S^{2 n-1} \stackrel{q \iota}{\longleftarrow} S^{2 n-1} .
$$

The indeterminacy is $q \pi_{2 n}(B U)=q \mathbb{Z} \subset \mathbb{Z}$, as $f$ has finite order. We often mod out by the indeterminacy and write $\left\{\zeta_{k}, f, q \iota\right\} \in \mathbb{Z} / q$. We can use these Toda brackets to establish the order of an element. Suppose, for example, that $16 f=0$, and we show that $\left\{\zeta_{k}, f, 16 \iota\right\} \in \mathbb{Z} / 16$ has order 16 . Then we can show $f$ must have order 16. By a Toda bracket identity, the image of $\left\{\zeta_{k}, f, 16 \iota\right\} \in \mathbb{Z} / 16$ under the projection $\mathbb{Z} / 16 \rightarrow \mathbb{Z} / 2$ is $1=\left\{\zeta_{k}, f 8 \iota, 2 \iota\right\} \in \mathbb{Z} / 2$. Hence $8 f=f 8 \iota$ must be nonzero. 
By the above, $r \in\left\{\zeta_{k}, f, q\right\}$ if and only if the following diagram homotopy commutes:

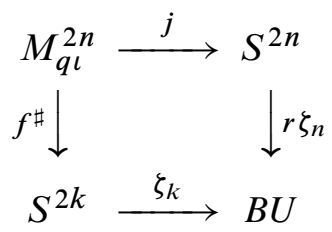

Adams defined the complex Adams operation $\Psi_{2}: B U \rightarrow B U$, which defines a ring homomorphism in $\tilde{K U}(X)=[X, B U]$ satisfying two properties: $\Psi_{2}\left(\zeta_{n}\right)=2^{n} \zeta_{n} \in$ $\pi_{2 n}(B U)$, and $\Psi_{2}(x)=x \cup x \in K U(X)(\bmod 2)$, for any class $x \in K U(X)$. Then $\left(\Psi_{2}-2^{k}\right) \zeta_{k}=0$, and we have the Toda bracket identity

$$
\left(\Psi_{2}-2^{k}\right)\left\{\zeta_{k}, f, q\right\}=\left\{\left(\Psi_{2}-2^{k}\right), \zeta_{k}, f\right\} q \in \pi_{2 n} B U \cong \mathbb{Z} .
$$

The Toda bracket $\left\{\left(\Psi_{2}-2^{k}\right), \zeta_{k}, f\right\}$ is essentially Adams's complex $e$-invariant, and it has indeterminacy $2^{k}$ times an odd number $\left(2^{n-k}-1\right.$, in fact). It easily follows from Adams [2], or the properties above, that $\left\{\left(\Psi_{2}-2^{k}\right), \zeta_{k}, v\right\}$ is $2^{k-2}$ times an odd number, for $k \geq 2$, and that $\left\{\left(\Psi_{2}-2^{k}\right), \zeta_{k}, \sigma\right\}$ is $2^{k-4}$ times an odd number, for $k \geq 4$. Then it follows from (13) that

Lemma 5.1 The Toda bracket $\left\{\zeta_{k}, \sigma, 16\right\} \in \mathbb{Z} / 16$ of the sequence

$$
B U \stackrel{\zeta_{k}}{\longleftarrow} S^{2 k} \stackrel{\sigma}{\leftarrow} S^{2 k+7} \stackrel{16}{\longleftarrow} S^{2 k+7}
$$

has order 16.

The Toda bracket $\left\{\zeta_{k}, v, 16\right\} \in \mathbb{Z} / 8$ of the sequence

$$
B U \stackrel{\zeta_{k}}{\longleftarrow} S^{2 k} \stackrel{v}{\leftarrow} S^{2 k+3} \stackrel{8}{\leftarrow} S^{2 k+3}
$$

has order 4.

Proof Choose $r \zeta_{k+4} \in\left\{\zeta_{k}, \sigma, 16\right\}$. Then for $a, b$ odd,

$$
2^{k} a r \zeta_{k+4}=\left(\Psi_{2}-2^{k}\right) r \zeta_{k+4}=\left\{\left(\Psi_{2}-2^{k}\right), \zeta_{k}, \sigma\right\} 16=2^{k-4} b 16 \zeta_{k+4}
$$

modulo the indeterminacy $2^{k+4}$, so $r$ is odd. $v$ is handled similarly.

Thus the composite (recall that $j \circ W=\sigma^{\sharp}$ )

$$
M_{16 \iota}^{2 k+8} \stackrel{W}{\rightarrow} M_{16 \iota}^{2 k} \stackrel{j}{\rightarrow} S^{2 k} \stackrel{\zeta_{k}}{\rightarrow} B U
$$

is homotopic to

$$
M_{16 \iota}^{2 k+8} \stackrel{j}{\rightarrow} S^{2 k+8} \stackrel{r \zeta_{k+4}}{\longrightarrow} B U
$$


where $r$ is odd. This is what is meant by saying that $W$ is a (2-local) $K$-theory isomorphism. Now suspend $S_{k} 7$ times:

$$
S^{8 k+7} \stackrel{i}{\rightarrow} M_{16 \iota}^{8 k+8} \stackrel{W^{\circ(k-2)}}{\longrightarrow} M_{16 \iota}^{24} \stackrel{\sigma^{\sharp}}{\rightarrow} S^{16} .
$$

By induction, the composite

$$
M_{16 \iota}^{8 k+8} \stackrel{W^{\circ(k-2)}}{\longrightarrow} M_{16 \iota}^{24} \stackrel{\sigma^{\sharp}}{\rightarrow} S^{16} \stackrel{\zeta_{8}}{\rightarrow} B U
$$

is homotopic to an odd multiple of the generator

$$
M_{16 \iota}^{8 k+8} \stackrel{j}{\rightarrow} S^{8 k+8} \stackrel{\zeta_{4 k+k}}{\longrightarrow} B U .
$$

By (12), $\left\{\zeta_{8}, S_{k}, 16 \iota\right\} \in \mathbb{Z} / 16$ is odd, and hence of order 16 . Thus $\left\{\zeta_{8}, S_{k} 8 \iota, 2 \iota\right\}=$ $1 \in \mathbb{Z} / 2$, and we have proved that $S_{k}$ has order 16 .

The case of $N_{k}$ is similar, but harder. By Lemma 5.1 and (12), the composite

$$
M_{8 \iota}^{12} \stackrel{\nu^{\sharp}}{\rightarrow} S^{8} \stackrel{\zeta_{4}}{\rightarrow} B U
$$

is homotopic to twice an odd multiple of

$$
M_{8 \iota}^{12} \stackrel{j}{\rightarrow} S^{12} \stackrel{\zeta_{6}}{\rightarrow} B U
$$

Suspend $N_{k} 3$ times:

$$
S^{8 k+3} \stackrel{i}{\rightarrow} M_{8 \iota}^{8 k+4} \stackrel{V^{\circ(k-1)}}{\longrightarrow} M_{8 \iota}^{12} \stackrel{\nu^{\sharp}}{\rightarrow} S^{8} .
$$

The composite

$$
M_{8 \iota}^{8 k+4} \stackrel{V^{\circ(k-1)}}{\longrightarrow} M_{8 \iota}^{12} \stackrel{\nu^{\sharp}}{\rightarrow} S^{8} \stackrel{\zeta_{4}}{\rightarrow} B U
$$

is homotopic to twice an odd multiple of

$$
M_{8 \iota}^{8 k+4} \stackrel{j}{\rightarrow} S^{8 k+4} \stackrel{\zeta_{4 k+2}}{\longrightarrow} B U
$$

and we conclude that $N_{k}$ has order at least 4 , which is good enough for our Poincaré embedding results.

Remark 5.2 Although the classes $N_{k}$ and $S_{k}$ were known to Mahowald, no proof of Theorem E is in the literature. See the appendix of Čadek and Crabb [7] for related computations. 
Proof of Corollary $\mathbf{F}$ For the Poincaré complexes defined using $N_{k}$, we consider the image of suspension homomorphism $E: \pi_{p}\left(S^{2}\right) \rightarrow \pi_{p+1}\left(S^{3}\right)$. The composite

$$
\pi_{p}\left(S^{3}\right) \stackrel{\eta_{*}}{\cong} \pi_{p}\left(S^{2}\right) \stackrel{E}{\rightarrow} \pi_{p+1}\left(S^{3}\right)
$$

coincides with $x \mapsto E \eta \cdot E x$ and $E \eta$ has order two. Hence, the image of $E: \pi_{p}\left(S^{2}\right) \rightarrow$ $\pi_{p+1}\left(S^{3}\right)$ is killed by 2 . By Proposition D, this gives the result, since $N_{k}$ has order 8 . (Alternatively, we could have used Selick [21], since $S^{3}$ has exponent 4.)

In the case of the Poincare complexes defined using $S_{k}$, we need to consider the image $E: \pi_{p}\left(S^{4}\right) \rightarrow \pi_{p+1}\left(S^{5}\right)$. By Selick's theorem, $S^{5}$ has exponent 8 , and therefore the image of $E$ is killed by 8 . Since $S_{k}$ has order 16, and the conclusion follows once again by application of Proposition D.

\section{Periodicity in high dimensional knot theory}

We show how Theorem A gives a homotopy-theoretic periodicity operator from $n-$ knots to $(n+4)-$ knots, inducing the four-fold periodicity in the knot cobordism groups (see Levine [17]). Knot periodicity has been geometrically described by Bredon [5], Cappell and Shaneson [8] and Kauffman [12].

Fix $n \geq 1$. By a (smooth) Seifert surface we mean an codimension one compact smooth submanifold $V^{n+1} \subset S^{n+2}$ in which $\partial V:=\Sigma^{n}$ is a homotopy $n$-sphere.

Two Seifert surfaces $V_{i} \subset S^{n+2}$ with $i=1,2$ are said to be equivalent if there is a diffeomorphism of $S^{n+2}$ which transfers $V_{1}$ to $V_{2}$.

Remark 6.1 If $\Sigma^{n} \subset S^{n+2}$ is a codimension two knot, then it has a Seifert surface. If the fundamental group of the complement of the knot is infinite cyclic, then there exists a Seifert surface for it which is simply connected (see Levine [16]). Conversely, if there is a 1-connected Seifert surface, then the complement has infinite cyclic fundamental group. One says in this instance that the knot is 1-simple.

\section{Homotopy Seifert surfaces}

Fix $n \geq 2$. A homotopy Seifert surface of dimension $n+1$ is a diagram of spaces

$$
S^{n} \stackrel{\alpha}{\longrightarrow} K \stackrel{p_{+}}{\underset{p_{-}}{\longrightarrow}} C
$$

in which

- $\alpha$ is an inclusion making $\left(K, S^{n}\right)$ into a Poincaré pair. 
- $K$ and $C$ are 1-connected and have the homotopy type of finite CW complexes;

- $p_{-} \circ \alpha=p_{+} \circ \alpha$;

- The homomorphism $\left(p_{+}\right)_{*}-\left(p_{-}\right)_{*}: H_{*}(K) \rightarrow H_{*}(C)$ is an isomorphism in positive degrees, where $H_{*}$ denotes singular homology.

(Compare Farber [10] and the paper by the author [19].) Denote these data by $\left(\alpha, p_{ \pm}\right)$. An equivalence $\left(\alpha, p_{ \pm}\right) \stackrel{\sim}{\rightarrow}\left(\alpha^{\prime}, p_{ \pm}^{\prime}\right)$ (with $\left.p_{ \pm}^{\prime}: K^{\prime} \rightarrow C^{\prime}\right)$ consists homotopy equivalences $a: K \rightarrow K^{\prime}$ and $b: C \rightarrow C^{\prime}$ such that $q_{ \pm} \circ a=b \circ p_{ \pm}$and $\alpha^{\prime}=a \circ \alpha$. Two homotopy Seifert surfaces will be called equivalent if there is a finite chain of equivalences connecting them.

Lemma 6.2 If $\left(\alpha, p_{ \pm}\right)$is a homotopy Seifert Surface, then $\Sigma \alpha$ is nullhomotopic. Furthermore, the homotopy class of the nullhomotopy is preferred.

Proof The map $\Sigma p_{+}-\Sigma p_{-}: \Sigma K \rightarrow \Sigma C$ is a homology isomorphism and therefore a homotopy equivalence by the Whitehead theorem. Call this map $h$. Then $h \circ \Sigma \alpha$ has a preferred nullhomotopy. The nullhomotopy for $\Sigma \alpha$ is now obtained by choosing a homotopy inverse for $h$.

\section{The relation between smooth Seifert and homotopy surfaces}

Let $V^{n+1} \subset S^{n+2}$ be a simply connected Seifert surface with $\partial V=\Sigma$. We will show how to construct an associated homotopy Seifert surface.

Fix an orientation preserving $\Sigma \cong S^{n}$ homeomorphism (here we are using the Poincaré conjecture). Choose a compact tubular neighborhood $U$ of $V$ and define $C$ to be the complement of the interior of $U$. Then $\partial U \subset C$. Identify $U$ with $V \times I$. Then $\partial U$ is identified with $V \times 0 \cup \Sigma^{n} \times I \cup V \times 1$.

Let $K_{-}:=V \times 0 \cup \Sigma^{n} \times[0,1 / 2]$ and $K_{+}=V \times 1 \cup \Sigma^{n} \times[1 / 2,1]$. Then $K_{-}$and $K_{+}$are homeomorphic by a preferred homeomorphism $h: K_{-} \rightarrow K_{+}$. Set $K:=K_{-}$and let $\alpha: S^{n} \rightarrow K$ be the identification $S^{n} \simeq \Sigma \times 1 / 2$ followed by the inclusion $\Sigma \times 1 / 2 \subset K_{-}$. Define $p_{-}: K \rightarrow C$ to be the inclusion, and $p_{+}: K \rightarrow C$ to be $h: K=K_{-} \rightarrow K_{+}$ followed by the inclusion $K_{+} \subset C$. By construction $p_{ \pm}$coequalize $\alpha$ and $\left(K, S^{n}\right)$ is a Poincaré pair. The homomorphism $\left(p_{+}\right)_{*}-\left(p_{-}\right)_{*}$ is seen to be an isomorphism in positive degrees using the pushout diagram

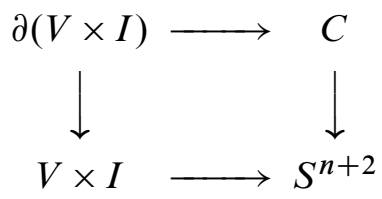


as follows: let $D_{0}$ be the result of removing the top cell of $\partial(V \times I)$. Then $D_{0}$ is identified with $K \vee K$ up to homotopy. With respect to this identification we have a homotopy pushout

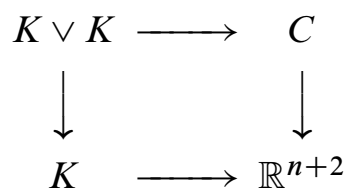

where $K \vee K \rightarrow K$ is the fold map and $K \vee K \rightarrow C$ is the map $\left(p_{-}, p_{+}\right)$. The conclusion now follows from the Mayer-Vietoris sequence for the pushout.

Theorem 6.3 Assume $n \geq 5$. Then the above induces a bijection between the set of equivalence classes of 1 -connected smooth Seifert surfaces in $S^{n+2}$ and the set of equivalence classes of homotopy Seifert surfaces of dimension $n+1$.

Proof (Existence) We need to show that the every homotopy Seifert surface arises up to equivalence from a smooth one. Let $\left(\alpha, p_{ \pm}\right)$be a homotopy Seifert surface, with $\alpha: S^{n} \rightarrow K$ and $p_{ \pm}: K \rightarrow C$. Let $D(K)$ denote the double mapping cylinder $K \times 0 \cup S^{n} \times I \cup K \times 1$, and let $p: D(K) \rightarrow C$ be the map defined by $p_{-}$on $K \times 0$, $p_{+}$on $K \times 1$ and the constant homotopy of the map $p_{-} \circ \alpha$ on $S^{n} \times I$. Without loss in generality, we can assume $p: D(K) \rightarrow C$ is a cofibration. Let

$$
N=(K \times I) \cup_{D(K)} C .
$$

Then $N$ has an orientation preserving homotopy equivalence to $S^{n+2}$. Furthermore, we have a Poincaré triad $(N ; K \times I, C ; D(K))$.

The scheme will be to use the diagram of smooth structure sets

$$
\begin{gathered}
\mathcal{S}(N ; K \times I, C ; D(K)) \stackrel{\Phi_{1}}{\cong} \mathcal{S}(N) \cong \mathcal{S}\left(S^{n+2}\right) \\
\mathcal{L}\left(K, S^{n}\right) \underset{\Phi_{2}}{\cong} \mathcal{S I} \\
\mathcal{S}(K \times I, D(K)) .
\end{gathered}
$$

The $h$ or $s$ decorations on the structure sets are unnecessary since we are in the simply connected case. Here, for an $n$-dimensional Poincaré pair $(X, \partial X)$ of 1 -connected complexes, $\mathcal{S}(X, \partial X)$ denotes the set generated by homotopy equivalences of pairs $(M, \partial M) \rightarrow(X, \partial X)$ subject to the relation of $h$-cobordism. Similarly, $\mathcal{S}(N ; K \times$ $I, C ; D(K))$ is the smooth structure set on the Poincaré triad $(N ; K \times I, C ; D(K))$. The function labeled $\Phi_{i}$ are forgetful maps, and $\Phi_{1}$ is an isomorphism by codimension one splitting (see Wall [24, Theorem 12.1]). The function labeled " $\times I$ " is given by taking cartesian product with the unit interval. It too is an isomorphism by the $\pi-\pi$ theorem [24, Theorem 3.3]. 
We proceed as follows. Choose the identity structure on $S^{n+2}$ and use the top isomorphism of the displayed diagram to give a smooth triad structure

$$
\left(S^{n+2} ; U, C^{\prime} ; \partial U\right) \stackrel{\sim}{\rightarrow}(N ; K \times I, C ; D(K))
$$

Then use the bottom isomorphism of the diagram to write $(U, \partial U)$ as $(V \times I, \partial(V \times I))$ up to diffeomorphism where $\Sigma:=\partial V$ is a homotopy $n$-sphere. Then we have a smooth triad $\left(S^{n+2} ; V \times I, C^{\prime}, \partial(V \times I)\right)$ yielding a smooth 1 -connected Seifert surface $V \times 1 / 2 \subset S^{n+2}$. It is clear that the homotopy Seifert surface associated with the smooth one is equivalent to the one we started with.

(Uniqueness) The proof will also appeal to the diagram appearing the proof of existence. Let $\left(\alpha, p_{ \pm}\right)$be a homotopy Seifert surface as above and suppose that $V_{i} \subset S^{n+2}$ are 1 -connected Seifert surfaces, $i=0$, 1, whose associated homotopy Seifert surfaces admit equivalences to $\left(\alpha, p_{ \pm}\right)$. The equivalences yield a pair of two smooth triad structures

$$
\left(S^{n+2} ; V_{i} \times I, C_{i}^{\prime}, \partial\left(V_{i} \times I\right)\right) \stackrel{\sim}{\rightarrow}(N ; K \times I, C ; D(K)),
$$

and by using the injectivity of $\Phi_{1}$, we infer that the two smooth triad structures are equivalent. We infer (by straightening $h$-cobordisms) that there is a diffeomorphism

$$
\psi:\left(S^{n+2} ; V_{0} \times I, C_{0}^{\prime}, \partial\left(V_{0} \times I\right)\right) \cong\left(S^{n+2} ; V_{1} \times I, C_{1}^{\prime}, \partial\left(V_{1} \times I\right)\right) .
$$

Using the injectivity of the function $\times I$, it follows that the restricted diffeomorphism $\psi: V_{0} \times I \rightarrow V_{1} \times I$ is pseudoisotopic to one of the form $\phi \times \mathrm{id}$, where $\phi: V_{0} \rightarrow V_{1}$ is a diffeomorphism. Choose such a pseudoisotopy and let

$$
H: \partial\left(V_{0} \times I\right) \times[0,1] \stackrel{\cong}{\rightarrow} \partial\left(V_{1} \times I\right) \times[0,1]
$$

be its restriction to the boundary. Choose collar neighborhoods $T_{i} \cong \partial\left(V_{i} \times I\right) \times[0,1]$ of $\partial\left(V_{i} \times I\right) \subset V_{i} \times I$. Then $H$ defines a diffeomorphism $T_{0} \cong T_{1}$ which extends to a diffeomorphism $H^{\prime}: V_{0} \times I \rightarrow V_{1} \times I$ by taking $\phi \times$ id on the complement of $T_{0}$. Extend $H^{\prime}$ to a diffeomorphism of $S^{n+2}$ using $\psi: C_{0}^{\prime} \rightarrow C_{1}^{\prime}$. The constructed diffeomorphism of $S^{n+2}$ takes $V_{0} \times 1$ to $V_{1} \times 1$, so we get an equivalence of between the smooth Seifert surfaces.

\section{Knot periodicity}

We define an operator which associates to a homotopy Seifert surface in dimension $n+1$ another one of dimension $n+5$.

Let $\left(\alpha, p_{ \pm}\right)$be a homotopy Seifert Surface of dimension $n+1$, where $\alpha: S^{n} \rightarrow K$ and $p_{ \pm}: K \rightarrow C$. Theorem A produces an attaching map $\beta: S^{n+4} \rightarrow \Sigma^{2} K$ whose 
cofiber satisfies Poincare duality. The proof shows that $\Sigma^{2} p_{-} \circ \beta$ and $\Sigma^{2} p_{+} \circ \beta$ are homotopic via a preferred homotopy $f: S^{n} \times I \rightarrow C$ (the verification of this is straightforward but tedious; we therefore omit it). The maps $\beta: S^{n+4} \rightarrow \Sigma^{2} K$ and $\Sigma^{2} p_{ \pm}: \Sigma^{2} K \rightarrow \Sigma^{2} C$ are close to defining a homotopy Seifert surface. However, there are two defects: (1) $p_{ \pm}$is only known to coequalize $\beta$ up to homotopy, and (2) $\beta$ is not a cofibration. We will show how to fix these problems.

Factor the map $\beta$ by a cofibration $\beta^{\prime}: S^{n} \rightarrow Z$ followed by a homotopy equivalence $h: Z \rightarrow \Sigma^{2} K$. Let $p_{ \pm}^{\prime}: Z \rightarrow C$ be $\Sigma^{2} p_{ \pm} \circ h$. Then $f$ defines a homotopy from $p_{-}^{\prime} \circ \beta^{\prime}$ to $p_{+}^{\prime} \circ \beta^{\prime}$. By the homotopy extension property, we obtain a map $q_{-}: Z \rightarrow C$ such that $q_{-} \circ \beta^{\prime}=\left(\Sigma^{2} p_{+}\right) \circ \beta^{\prime}$. Set $q_{+}=\Sigma^{2} p_{+}$. Then $\left(\beta^{\prime}, q_{ \pm}\right)$is a homotopy Seifert surface.

We now sketch a proof that the assignment $\left(\alpha, p_{ \pm}\right) \mapsto\left(\beta^{\prime}, q_{ \pm}\right)$yields four-fold periodicity in knot cobordism. Although the verification is somewhat tedious, the basic idea is that the intersection pairing of $X=K \cup_{\alpha} D^{n+1}$ together with the homomorphism that $p_{+}$induces on homology completely determines the smooth knot cobordism class of $\left(\alpha, p_{ \pm}\right)$(here we are implicitly using Theorem 6.3 to identify $\left(\alpha, p_{ \pm}\right)$with a smooth Seifert surface to make sense of the smooth knot cobordism class of the homotopy Seifert surface). Then the result is established once we show that the intersection pairing of $Y:=\left(\Sigma^{2} K\right) \cup_{\beta} D^{n+5}$ has the same intersection pairing as $X$ up to regrading (since $q_{ \pm}$and $p_{ \pm}$induce the same homomorphisms on homology). That is idea. Some details follow.

Note that the basepoint for $S^{n}$ gives basepoints for $K$ and $C$. The maps $D(K) \rightarrow$ $K \times I \rightarrow K$ and $D(K) \rightarrow C$ combine to a give a map $D(K) \rightarrow K \times C$, which we follow up with the quotient map $K \times C \rightarrow K \wedge C$ to obtain a map $D(K) \rightarrow K \wedge C$. The commutative diagram

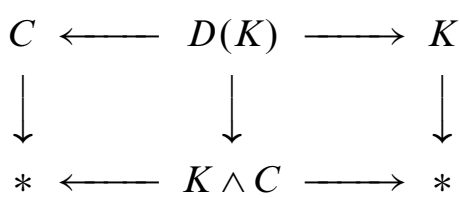

induces a map of homotopy pushouts $d: S^{n+2} \rightarrow \Sigma K \wedge C$ which is an $S$-duality map, which in turn yields the Alexander duality isomorphism $H_{*}(K) \cong H^{n+1-*}(C)$ in positive degrees. Then the homology class $d_{*}\left(\left[S^{n+1}\right]\right) \in H_{n+1}(K \wedge C)$ determines a class $d^{\sharp} \in H^{n+1}(K \wedge C)$ which are Alexander dual via the duality map $d \wedge d$. Let

$$
\delta: H_{j}(K) \otimes H_{n+1-j}(C) \rightarrow \mathbb{Z}
$$

be given by $\delta(a \otimes b)=d^{\sharp}(a \times b)$. Then $\delta$ is the Alexander pairing. 


\section{Definition 6.4 The Seifert pairing}

$$
\Phi: H_{j}(K) \otimes H_{n+1-j}(K) \rightarrow \mathbb{Z}
$$

of $\left(\alpha, p_{ \pm}\right)$is given by $\left.\Phi(x \otimes y)=\delta\left(\left(p_{+}\right)_{*}(x) \otimes y\right)\right)$.

To establish periodicity, it will be enough by Levine [17] to show that that the Seifert pairings for $\left(\alpha, p_{ \pm}\right)$and $\left(\beta^{\prime}, q_{ \pm}\right)$coincide. To keep the discussion simple, we will only verify this when $K$ is a suspension (this is sufficient because Levine showed that every smooth $n$-knot is cobordant to one having a Seifert surface which is $\lfloor(n+1) / 2\rfloor-$ connected (see Levine [16]), and such Seifert surfaces desuspend by the Freudenthal theorem). We may therefore assume that $K$ is $\lfloor(n+1) / 2\rfloor$-connected, $K$ is a suspension $\Sigma L$, and $C$ is also identified with $\Sigma L$ using $p_{+}-p_{-}: \Sigma L \rightarrow C$. Then map $\alpha: S^{n} \rightarrow \Sigma L$ factors as

$$
S^{n} \stackrel{\widehat{\alpha}}{\rightarrow} \Sigma L \wedge L \stackrel{P}{\rightarrow} \Sigma L
$$

where $P$ is the Whitehead product. Furthermore, the composite

$$
S^{n+1} \stackrel{\Sigma \widehat{\alpha}}{\longrightarrow} \Sigma L \wedge \Sigma L \stackrel{1+\tau_{\Sigma L}}{\longrightarrow} \Sigma L \wedge \Sigma L
$$

is an $S$-duality map. Likewise, the proof of Theorem A shows that the attaching map $\beta: S^{n+4} \rightarrow \Sigma^{2} L$ is given by

$$
S^{n+4} \stackrel{\Sigma^{4} \hat{\alpha}}{\longrightarrow} \Sigma\left(\Sigma^{2} L\right) \wedge\left(\Sigma^{2} L\right) \stackrel{P}{\rightarrow} \Sigma\left(\Sigma^{2} L\right) .
$$

It is clear from this description that the intersection pairings for $X^{n+1}=K \cup_{\alpha} D^{n+1}$ and $Y=\Sigma^{2} K \cup_{\beta} D^{n+5}$ coincide after regrading, since the cup product structure of $X$ is completely determined by the homomorphism induced by $\phi:=\left(1+\tau_{\Sigma L}\right) \circ(\Sigma \hat{\alpha})$ on homology. More precisely, by Boardman and Steer [4], there is a homotopy commutative diagram

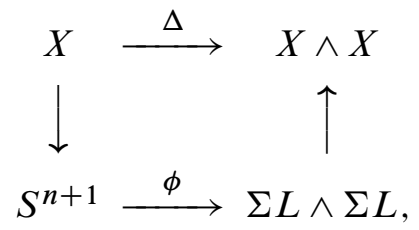

where $\Delta$ is the diagonal (inducing the cup product), the left vertical map is the pinch map onto the top cell, and the right vertical map is the inclusion.

Notice the inclusion $K \rightarrow X$ induces an isomorphism in homology in degrees $\neq n+1$. Furthermore there is a map $\Sigma X \rightarrow \Sigma C$ which is a homology isomorphism in degrees $\neq n+1$. The latter map is defined as follows: the Poincaré embedding gives an equivalence between $\Sigma X$ with the cofiber $S^{n+2} \cup \operatorname{Cone}(C)$; compose this equivalence with the connecting map $S^{n+2} \cup \operatorname{Cone}(C) \rightarrow \Sigma C$ appearing in Barratt-Puppe sequence. 
(In terms of the splitting, $\Sigma X \simeq \Sigma K \vee S^{n+2}$, the restriction of the map $\Sigma X \rightarrow \Sigma C$ to $\Sigma K$ is identified with the homotopy equivalence $\Sigma p_{+}-\Sigma p_{-}$, whereas the restriction to the $S^{n+2}$ summand is trivial.)

Thus the intersection pairing of $X$ can be rewritten in positive degrees as

$$
H_{j}(X) \otimes H_{n+1-j}(X) \cong H_{j}(K) \otimes H_{n+1-j}(C) \rightarrow \mathbb{Z},
$$

where the second homomorphism is the Alexander pairing $\delta$. Thus, the intersection pairing of $X$ and the Alexander pairing of the Poincare embedding associated with $\left(\alpha, p_{ \pm}\right)$coincide in positive degrees. A similar statement holds for $Y$.

Since the intersection pairings for $X$ and $Y$ coincide (after regrading), the Alexander pairings arising from $\left(\alpha, p_{ \pm}\right)$and $\left(\beta^{\prime}, q_{ \pm}\right)$also coincide. Since $q_{ \pm}$coincides with $p_{ \pm}$on homology, the Seifert pairings of $\left(\alpha, p_{ \pm}\right)$and $\left(\beta^{\prime}, q_{ \pm}\right)$coincide.

\section{The period of a finite complex}

Theorem A is not the most general result. If $X=\mathbb{R} P^{3}$, then the spine of $X$ is $\mathbb{R} P^{2}$, and the top cell of $X$ splits off after two suspensions but not one. On the other hand, $\Sigma^{2} \mathbb{R} P^{2}$ is the spine of $V_{2}\left(\mathbb{R}^{5}\right)$, the Stiefel manifold of 2 -frames in $\mathbb{R}^{5}$.

If $X^{n}$ is a Poincare complex with spine $K$ such that the top cell of $X$ splits off after one suspension, then Theorem A can be iterated to produce a sequence of Poincaré complexes $Y_{j}$ of dimension $n+4 j$ having spine $\Sigma^{2 j} K$. In this way, we obtain a periodic family of Poincaré complexes. This motivates

Definition 7.1 A finite complex $K$ is said to be $j$-periodic for some positive integer $j$ if there is an integer $c$ and a sequence of Poincaré complexes $X_{1}, X_{2}, \ldots$ such that the spine of $X_{i}$ is $\Sigma^{c+i j} K$. If $K$ is $j$-periodic for some $j$, we say that $K$ is periodic. If $K$ is not periodic, we declare it to be aperiodic.

The period of $K$, denoted period $(K)$, is the smallest positive integer $r$ such that $K$ is $r$-periodic. If there is no such $r$, then we write period $(K)=\infty$.

(1) If $K$ is periodic, then $K$ is self Spanier-Whitehead dual. This is a direct consequence of Proposition 2.2 below.

(2) $\operatorname{period}\left(S^{k}\right)=\infty$, since there are only a finite number of Hopf invariant one elements. 
(3) period $\left(\mathbb{R} P^{2}\right) \leq 2$, because the spine of the Stiefel manifold $V_{2}\left(\mathbb{R}^{3+2 i}\right)$ (consisting of two-frames in $\mathbb{R}^{3+2 i}$ ) is $\Sigma^{2 i} \mathbb{R} P^{2}$. Furthermore, $\Sigma \mathbb{R} P^{2}$ is the spine of $\mathrm{SU}(3) / \mathrm{SO}(3)$, but Mahowald has pointed out to us that this is the only odd suspension of $\mathbb{R} P^{2}$ which is the spine of a Poincaré complex. So, period $\left(\mathbb{R} P^{2}\right)=2$.

(4) If $K$ is the spine of a Poincaré complex which embeds in codimension one, then Theorem B shows period $(K)=1$.

(5) If $K$ is the spine of a Poincare complex whose top cell splits off after a single suspension, then period $(K) \leq 2$, by Theorem A.

(6) Let $K$ be the spine of a $4 k$-dimensional Poincaré complex $X$, such that the Euler characteristic $\chi(K)$ is even. Then $\Sigma K$ cannot be the spine of a Poincaré complex of dimension $4 k+2$ by Example 1.1. Hence, $\operatorname{period}(K)>1$.

(7) If $K=\operatorname{spine}(X)$ and $L=\operatorname{spine}(Y)$ for Poincaré complexes $X^{n}$ and $Y^{n}$, then $K \vee L$ is periodic and period $(K \vee L) \leq \operatorname{lcm}(\operatorname{period}(K), \operatorname{period}(L))$. To see this, set $r=\operatorname{period}(K)$ and set $s=\operatorname{period}(L)$. Let $\ell$ denote their least common multiple. Define $Z_{i}:=X_{(i \ell) / r} \# Y_{(i \ell) / s}$, where $X_{i}$ has spine $\Sigma^{i r} K$ and $Y_{i}$ has spine $\Sigma^{i s} K$. Then $Z_{i}$ has spine $\Sigma^{i \ell}(K \vee L)$. Equality generally fails: for example, $\operatorname{period}\left(S^{p}\right)=\infty=\operatorname{period}\left(S^{q}\right)$, but $\operatorname{period}\left(S^{p} \vee S^{q}\right)=1$.

Our notion of periodicity is linear, in that the gaps between the number of suspensions of $K$ appearing in the definition is constant. The following, due to Mahowald (private communication), is an example of a 2-cell complex which exhibits exponential periodicity, in the sense that the gaps grow at an exponential rate.

Theorem (Mahowald) Let $K=\mathbb{H} P^{2}$ be the homotopy cofiber of the $v: S^{7} \rightarrow S^{4}$. Let $\delta(i)=2^{i+2}$. Then $\Sigma^{\delta(i)} K$ is a spine of a Poincaré complex for $i>0$. Furthermore, one cannot fill in the gaps: if $\Sigma^{j} K$ is the spine of a Poincaré complex, for some $j>0$, then $j=2^{i+2}$ for some $i$.

We ask a final question: When is a finite complex periodic?

\section{References}

[1] J F Adams, On the non-existence of elements of Hopf invariant one, Ann. of Math. (2) 72 (1960) 20-104 MR0141119

[2] J F Adams, On the groups $J(X) I V$, Topology 5 (1966) 21-71 MR0198470

[3] W D Barcus, M G Barratt, On the homotopy classification of the extensions of a fixed map, Trans. Amer. Math. Soc. 88 (1958) 57-74 MR0097060 
[4] J M Boardman, B Steer, On Hopf invariants, Comment. Math. Helv. 42 (1967) 180221 MR0221503

[5] G E Bredon, Regular $\mathbb{O}(n)$-manifolds, suspension of knots, and knot periodicity, Bull. Amer. Math. Soc. 79 (1973) 87-91 MR0310901

[6] W Browder, Embedding smooth manifolds, from: "Proc Internat. Congr. Math. (Moscow, 1966)", Izdat. "Mir”, Moscow (1968) 712-719 MR0238335

[7] M Čadek, M Crabb, G-structures on spheres, Proc. London Math. Soc. (3) 93 (2006) 791-816 MR2266967

[8] S E Cappell, J L Shaneson, Topological knots and knot cobordism, Topology 12 (1973) 33-40 MR0321099

[9] F R Cohen, A course in some aspects of classical homotopy theory, from: "Algebraic topology (Seattle, Wash., 1985)”, Lecture Notes in Math. 1286, Springer, Berlin (1987) 1-92 MR922923

[10] MŠ Farber, Isotopy types of knots of codimension two, Trans. Amer. Math. Soc. 261 (1980) 185-209 MR576871

[11] J P E Hodgson, Questions appearing in the Problem Session, from: "Proceedings of the Northwestern Homotopy Theory Conference (1982)", Contemp. Math. 19 (1983) 448

[12] L H Kauffman, Branched coverings, open books and knot periodicity, Topology 13 (1974) 143-160 MR0375337

[13] J R Klein, On the homotopy embeddability of complexes in Euclidean space I: The weak thickening theorem, Math. Z. 213 (1993) 145-161 MR1217676

[14] J R Klein, Poincaré duality embeddings and fiberwise homotopy theory, Topology 38 (1999) 597-620 MR1670412

[15] A A Kosinski, Differential manifolds, Pure and Applied Mathematics 138, Academic Press, Boston (1993) MR1190010

[16] J Levine, Unknotting spheres in codimension two, Topology 4 (1965) 9-16 MR0179803

[17] J Levine, Knot cobordism groups in codimension two, Comment. Math. Helv. 44 (1969) 229-244 MR0246314

[18] M Mahowald, The image of $J$ in the EHP sequence, Ann. of Math. (2) 116 (1982) 65-112 MR662118

[19] W Richter, High-dimensional knots with $\pi_{1} \cong \mathbb{Z}$ are determined by their complements in one more dimension than Farber's range, Proc. Amer. Math. Soc. 120 (1994) 285294 MR1195730

[20] W Richter, A homotopy-theoretic proof of Williams's metastable Poincaré embedding theorem, Duke Math. J. 88 (1997) 435-447 MR1455528 
[21] P Selick, 2-primary exponents for the homotopy groups of spheres, Topology 23 (1984) 97-99 MR721456

[22] H Toda, Composition methods in homotopy groups of spheres, Annals of Mathematics Studies 49, Princeton University Press, Princeton, N.J. (1962) MR0143217

[23] C T C Wall, Poincaré complexes I, Ann. of Math. (2) 86 (1967) 213-245 MR0217791

[24] C T C Wall, Surgery on compact manifolds, London Mathematical Society Monographs 1, Academic Press, London (1970) MR0431216

[25] G W Whitehead, Elements of homotopy theory, Graduate Texts in Mathematics 61, Springer, New York (1978) MR516508

Department of Mathematics, Wayne State University

Detroit MI 48202, USA

Department of Mathematics, Northwestern University

Evanston IL 60208, USA

klein@math.wayne.edu, richter@math.northwestern.edu

Received: 8 July 2007 Revised: 7 March 2011 